\title{
Rapid identification of mycobacteria from AIDS patients by capillary electrophoretic profiling of amplified SOD gene
}

\author{
T J Bull, D C Shanson, L C Archard
}

\begin{abstract}
Aim-Rapid differentiation of mycobacterial species at the genomic level.

Methods-The manganese superoxide dismutase (SOD) gene (464 bp) and 16SrRNA (353 bp) from 104 isolates (18 species) of mycobacteria were amplified using polymerase chain reaction (PCR). Products were sequenced and a phenogram of SOD sequences derived. PCR products of SOD gene were digested with HaeIII, and restriction fragment profiles visualised using capillary electrophoresis.
\end{abstract}

Results-Novel SOD sequences were found for M szulgai, M marinum, M phlei, $M$ smegmatis, M chelonei, M paratuberculosis, M malmoense, M intracellulare serotype $7, M$ intracellulare serotype 18 , and $M$ celatum types 1,2 , and 3 . Phylogenetic analysis indicated that 18 of 19 species studied had 8-29\% interspecies and $<6 \%$ intraspecies sequence diversity in the SOD gene. No consistent differences were detected between AIDS and non-AIDS isolates. $M$ paratuberculosis showed a unique SOD sequence with a $1 \cdot 1 \%$ (SD $0.5 \%$ ) diversity from $M$ avium. Capillary electrophoresis profiles were able to differentiate 16 of 18 species within 24 hours.

Conclusions-A phenogram of SOD sequences clearly delineated all mycobacterial species and showed two distinct clusters, fast growing species, and the $M a v-$ ium complex (MAC). Within the MAC, $M$ avium (five types), $M$ intracellulare (five types), $M$ scrofulaceum (two types), and $M$ paratuberculosis (one type) could be demonstrated. Phylogenetic diversity of $M$ celatum from MAC, previously suggested by 16 SrRNA data, was confirmed. This simple and rapid method for DNA extraction, in conjunction with capillary electrophoresis of SOD restriction fragments, allows rapid identification of mycobacterial isolates.

(f Clin Pathol: Mol Pathol 1995;48:M124-M132)

Keywords: Mycobacteria, superoxide dismutase, rapid identification.
Until recently the taxonomic classification and identification of bacteria has been restricted to phenotypic systems based upon biochemical tests. ${ }^{1}$ The advent of molecular based techniques, which can rapidly detect and identify bacteria in clinical samples, ${ }^{2-4}$ has highlighted the need for a genetically based classification of bacteria. The relatively slow growth of mycobacteria in culture media (1-12 weeks) has emphasised this need, with particular reference to the rapid differential diagnosis of $M$ tuberculosis and $M$ avium complex (MAC) infection in AIDS patients. ${ }^{5-7}$ Studies have shown the short variable region of the $16 \mathrm{SrRNA}$ gene to be suitable for phylogenetic comparisons ${ }^{8}$ and a detailed taxonomic system has been proposed for the Mycobacteriaceae based upon these data. ${ }^{9}$ Confirmation of these relationships in other genes has not been made; however, recent studies of the manganese superoxide dismutase (SOD) gene have indicated a significant sequence diversity, ${ }^{10}$ suitable for phylogenetic analysis.

\section{Methods}

CULTURES

The 104 isolates used for this study were all identified by conventional biochemical means at the Regional Centre for Tuberculosis Bacteriology, PHLS, Dulwich Hospital, London, and consisted of: (1) 52 isolates of MAC from 36 patients (this group contained 35 isolates of $M$ avium from 23 patients; 10 identical isolates from eight patients described here as $M$ celatum type 3; four isolates of $M$ intracellulare, serotype unknown, from two patients; and three isolates of $M$ intracellulare from two patients consistent with serotype 18), 10 isolates of $M$ kansasii from eight patients, nine isolates of $M$ tuberculosis from nine patients, one isolate of $M$ scrofulaceum, and one isolate of $M$ simiae, all from patients with AIDS; (2) 10 isolates of MAC from 10 patients, one isolate of $M$ malmoense, two isolates of $M$ kansasii, all from patients without AIDS; (3) 18 type culture mycobacterial reference strains: $M$ fortuitum (NCTC 10394), $M$ smegmatis (NCTC 10265), $M$ phlei (NCTC 8151), $M$ \\ Medical Microbiology
}


szulgai (NCTC 25932), M tuberculosis H37Rv (NCTC 7416), $M$ malmoense (NCTC 11298), $M$ avium (NCTC 8559), $M$ intracellulare (NCTC 10682), $M$ chelonei (NCTC 0946), $M$ xenopi (NCTC 10042), $M$ marinum (NCTC 2275), M gordonae (NCTC 10267), M kansasii (NCTC 10268), $M$ scrofulaceum (NCTC 10803), $M$ paratuberculosis (NCTC 8578), $M$ intracellulare serotype 7 (ATCC 35847), $M$ celatum type 1 (ATCC 51131), and $M$ celatum type 2 (ATCC 51130).

\section{DNA EXTRACTION}

All cultures were grown to purity on Middlebrooks $7 \mathrm{H} 10$ for $4-6$ weeks. Two or three colonies were emulsified in $500 \mu \mathrm{l} \mathrm{TE}$ buffer (50 mM Tris (pH 8.2), $1 \mathrm{mM}$ EDTA) in a screw capped reaction tube and centrifuged at $13000 \mathrm{rpm}$ for five minutes. The supernatant was removed and the pellet resuspended in $25 \mu \mathrm{l}$ TE. This suspension was frozen rapidly for 10 seconds in liquid nitrogen and then sonicated in a water bath (Perkin Elmer) for 30 seconds at room temperature. This procedure was repeated three times and the suspension heated for five minutes at $98^{\circ} \mathrm{C}$ in a heating block, then rapidly cooled for $10 \mathrm{sec}-$ onds in liquid nitrogen and sonicated again for one minute. DNA preparations were stored at $-20^{\circ} \mathrm{C}$ before amplification.

\section{PRIMERS}

For SOD

Primers were designed from homologous regions of previously published SOD gene sequences from $M$ tuberculosis $^{7}$ and $M$ leprae $^{11}$ to give a 464 base pair (bp) product. These were as follows:

\section{SF1: ACATCTCGGGTCAGATCAACGACG} SR1: GACGTTCTTGTACTGCAGGTA

\section{For $16 \operatorname{Sr} R N A$}

Primers were as previously described ${ }^{3}$ and gave a 353 bp product. These were as follows:

\section{SpA: AGAGTTTGATCCTGGCTCAG} 16SPC*: CCCACTGCTGCCTCCCGTAG

Primers were synthesised on an Applied Biosystems 380B automated synthesiser and desalted using NAP 10 columns (Pharmacia). Primer quality was assessed following capillary electrophoresis using Microgel capillaries (Applied Biosystems) on an Applied Biosystems 270A capillary electrophoresis unit.

\section{PCR CONDITIONS AND SEQUENCING}

PCR reaction mixes $(100 \mu \mathrm{l})$ for each of the primer pairs were prepared from each of the test isolates using the following conditions: $1 \mu \mathrm{l}$ of extracted chromosomal DNA was amplified in a Gene Amp 9600 thermocycler (Perkin Elmer) with $10 \mu \mathrm{l} 10 \mathrm{X}$ polymerase chain reaction (PCR) buffer (100 mM Tris (pH 8.3), $500 \mathrm{mM} \mathrm{KCl}, 15 \mathrm{mM} \mathrm{MgCl} 2,0.015 \%$ gelatin). $16 \mu \mathrm{l}$ deoxyribonucleotide $\mathrm{mix}(1 \cdot 25 \mu \mathrm{mol}$ per
$\mathrm{dNTP}), 5 \mu \mathrm{l}$ of each primer $(20 \mu \mathrm{M})$, and $5 \mathrm{U}$ Taq polymerase made to $100 \mu \mathrm{l}$ with water. The cycling profile consisted 30 cycles of one minute at $94^{\circ} \mathrm{C}$, one minute at $58^{\circ} \mathrm{C}$, and one minute at $72^{\circ} \mathrm{C}$, followed by a final incubation at $72^{\circ} \mathrm{C}$ for five minutes. Relevant products were then isolated by agarose gel electrophoresis of $70 \mu \mathrm{l}$ amplified reaction product, excised by use of a sterile scalpel, extracted with phenol-chloroform-isoamylalcohol, concentrated by ethanol precipitation, and final concentration estimated for sequencing using a further agarose gel electrophoresis. Automated sequencing was made, with $0.5 \mu \mathrm{g}$ product DNA, using the PRISM Taq dye termination system (Perkin Elmer) with the original primers. The cycling profile for sequencing was 25 cycles of $96^{\circ} \mathrm{C}$ for 15 seconds followed by $60^{\circ} \mathrm{C}$ for five minutes. Sequencing of both strands of product was made and aligned for base verification. Sequences with ambiguities between strands were checked by repeat sequencing from the same DNA preparation.

\section{RESTRICTION DIGESTION AND CAPILLARY}

ELECTROPHORESIS

The PCR reaction mix $(8 \mu \mathrm{l})$ was used for restriction digestion by mixing with $1 \mu \mathrm{l}(1 \mathrm{U} /$ $\mu \mathrm{l})$ HaeIII enzyme (Promega) and $1 \mu$ OnePhorAll buffer (Pharmacia). Digestions were made at $37^{\circ} \mathrm{C}$ for 1.5 hours. Samples for capillary electrophoresis were prepared by mixing $1 \mu \mathrm{l}$ sample digest, $18 \mu \mathrm{l}$ water, and $1 \mu \mathrm{l}(38 \mu \mathrm{g} /$ ml) standard pBR322 HaeIII digest (Sigma). Samples were electrokinetically loaded for 80 seconds at $-5 \mathrm{kV}$ and then electrophoresed for 15 minutes at $-13 \mathrm{kV}$. Capillary electrophoresis profiles were obtained using a DNA fragment analysis kit (Applied Biosystems) according to the manufacturers' instructions on an Applied Biosystems 270HT interfaced with Turbochrom integration software (Perkin Elmer). An estimate of restriction fragment sizes was then calculated by calibrating retention times of the internal standard against sample fragments.

\section{PHYLOGENETIC ANALYSIS}

Phylogenetic analyses were made using the PHYLIP package available on SEQNET using the FITCH algorithm and Jukes and Cantor DNADIST method $^{12}$ for estimations of phylogenetic diversity. Human SOD sequence ${ }^{13}$ was aligned to mycobacterial sequences and used as an outgroup to root the tree. Values are given as means (SD).

\section{Results}

BIOCHEMICAL IDENTIFICATION AND 16SrRNA PROFILES

A 16SrRNA sequence profile was obtained from all 104 isolates. In the majority of isolates the profile corresponded to the biochemical identification and previously reported 16SrRNA profiles ${ }^{3}$ after sequencing one strand only. Isolates giving irregular sequences were verified from both strands and repeated if 
193

human CACCACAGCAAGCACCACGCGGCCTACGTGAACAACCTGAACGTCACCGAGGAGAAGTACCAGGAGGCGTTGGCCAAGGGAGATGTTACAGCCCAGACAG avium typel CACCACACCAAGCACCACGCCACCTACGTCAAAGGCGTGAACGACGCTCTTGCCAAGCTCGAAGAGGCCCGCGCCAACGAGGACCACGCTGCG-ATCTTC av1 um type 2 CACCACACCAAGCACCACGCCACCTACGTCAAAGGCGTGAACGACGCTCTTGCCAAGCTCGAAGAGGCCCGCGCCAACGAGGACCACGCTGCG-ATCTTC avi um type3 CACCACACCAAGCACCACGCCACCTACGTCAAAGGCGTGAACGACGCTCTTGCCAAGCTCGAGGAGGCCCGCGCCAACGAGGACCACGCTGCG-ATCTTC av 1 um type 4 CACCACACCAAGCACCACGCCACCTACGTCAAAGGCGTGAACGACGCTCTTGCCAAGCTCGAAGAGGCCCGCGCCAACGAGGACCACGCTGCG-ATCTTC avlum type 5 CACCACACCAAGCACCACGCCACCTACGTCAAAGGCGTGAACGACGCTCTTGCCAAGCTCGAAGAGGCCCGCGCCAACGAGGACCACGCTGCG-ATCTTC celat um t 3 CACCACAGCAAACACCATGCGACCTACGTGAAGGGCGCCAACGACGCGCTCGAAAAACTCGAGGAAGCACGCGCCAAGGACGACCAGTCGACC-ATCCTC Celactict celatum.t2 CACCACAGCAAACACCATGCGACCTACGTGAAGGGCGCGAACGACGCGCTTGAAAAACTCGAGGAAGCACGCGCCAAGGACGACCAGTCGACC-GTCCTG

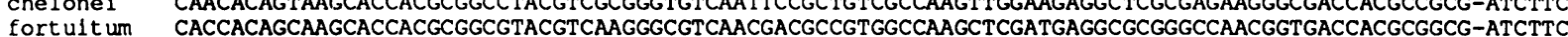
fort uit um CACCACAGCAAGCACCACGCGGCGTACGTCAAGGGCGTCAACGACGCCGTGGCCAAGCTCGATGAGGCGCGGGCCAACGGTGACCACGCGGCG-ATCTTC gordonae TCACACAGTAAGCACCACGCCACCTACGTCAAAGGCGTCAACGACGCGGTCGCCAAGCTGGAAGAAGCGCGCGCCAAAGGGACCACTCGGCC-ATCTTT int ra type 1 CACCACAGTAAGCACCACGCCACCTACGTCAAAGGCGTGAACGACGCTCTGTCCAAGCTCGAAGAGGCCCGTGCCAACGAAGATCACGCTGCG-ATCTTC int ra type 2 CACCACAGTAAGCACCACGCCACCTACGTCAAAGGCGTGAACGACGCTCTGTCCAAGCTCGAAGAGGCCCGTGCCAACGAAGATCACGCTGCG-ATCTTC int ra type 3 TCACACAGTAAGCACCACGCCACCTACGTCAAAGGCGTGAACGACGCTCTGTCCAAGCTCGAAGAGGCCCGTGCCAACGAAGATCACGCTGCG-ATCTTC Int ra type 4 TACCACAGTAAGCACCACGCCACATACGTCAAAGGCGTGAATGACGCTCTGTCCAAGCTCGAAGAGGCCCGCGCCAACGAGGACCACGCTGCG-ATCTTC intra type5 CACCACACTAAGCACCACGCCACGTACGTCAAAGGCGTGAACGACGCTCTGTCCAAGCTCGAAGAGGCCCGCGCCAACGAGGACCACGCTGCG-ATCTTC leprae CACCACACCAAGCACCACGCCGCATATGTCAAAGGTGTCAATGACGCGCTTGCCAAACTTGACGAGGCACGCGCCAAAGACGACCACTCCGCG-ATTTTT malmoense CACCACAGCAAGCACCACGCCGCCTACGTCAAGGGCGTGAACGACGCCGTCGCCAAGCTTGAAGAGGCGCGGGCCAAGGACGACCACTCGGCG-ATCTTC marinum TCACACAGTAAGCACCACGCCACCTACGTCAAGGGTGCCAATGACGCCGTCACCAAACTCGAGGAAGCGCGCGCTAAGGAAGACCACTCGACG-ATCCTG kansas 11 t 1 CACCACAGTAAGCACCACGCCACCTACGTCAAGGGCGCCAACGATGCGGTCGCCAAACTCGAAGAGGCGCGCGCCAAGGAAGACCACTCGGCG-ATCTTG kansas 11 t 2 GCACACAGTAAGCACCACGCCACCTACGTCAAGGGCGCCAACGATGCGGTCGCCAAACTCGAAGAGGCGCGCGCCAAGGAAGACCACTCGGCG-ATCTTG paratb CACCACACCAAGCACCACGCCACCTACGTCAAAGGCGTGAACGACGCTCTTGCCAAGCTCGAAGAGGCCCGCGCCAACGAGGACCACGCTGCG-ATCTTC M.sp.40407 TCACACAGCAAACACCATGCGACCTACGTCAAGGGTGCCAACGACGCGCTCGCCAAGCTCGAGGAGGCGCGCGCCAACGATGACCAGTCGACG-ATCCTG scrof t1 CATCACACCAAGCACCACGCCACGTACGTCAAGGGCGTGAACGACGCCGTCGCCAAACTCCAAGAGGCACGCGCCAATGACGACCACGCCGCG-ATCTTC scrof t 2 CATCACACCAAGCACCACGCCACCTACGTCAAGGGCGTGAACGACGCGGTCGCCAAACTCCAAGAGGCGCGCGCCAACGACGACCACGCCGCG-ATCTTC simiae smegmat is szulgai CACCACAGCAAGCACCATGCGACGTACGTCAAGGGTTTGAACGACGCCATTGCCAAGCTTGAAGAGGCACGGGCCAACGACGACCACGCCGCG-ATCTTC CACCACAGCAAGCACCACGCGACCTACGTCAAGGGTGTGAACGACGCGATTGCCAAAGCTCGAGGAGGCACGGGCCAACGGTGACCACGCGGCC-ATCTTC TCACACAGCAAGCACCACGCCACCTACGTCAAGGGCGCCAATGACGCTGTCGCCAAACTCGAGGAGGCGCGCGCTCAGGAGGACTTTTCGTCG-ATCTTC CACCACAGCAAGCACCACGCGACGTACGTCAAAGGCGCCAACGACGCGCTCGCCAAGCTGGAGGAGGCGCGCGCCAAAGACGATCATTCCGCG-ATCGTC 293

xenop1

human CTCTTCAGCCTGCAC-TGAAGTTCAATGGTGGTGGTCATATCAATCATAGCATTTTCTGGACAAACCTCAGCCCTAACGGTGGTGGAGAACCCAAAGGGG av lum type 1 CTGAACGAAAAGAACCTCGCCTTCCACCTGGGCGGCCACGTCAACCACTCGATCTGCTGGAAGAACCTCTCCCCGCACGCCCGTGACAAGCCCACCGGTG av1um type 2 CTGAACGAAAAGAACCTCGCCTTCCACCTGGGCGGCCACGTCAACCACTCGATCTGGTGGAAGAACCTGTCGCCGGACGGCGGTGACAAGCCCACCGGTG av 1 um type 3 CTGAACGAAAAGAACCTCGCCTTCCAGCTGGGCGGCCACGTCAACCACTCGATCTGGTGGAAGAACCTGTCGCCGGACGGCGGTGACAAGCCCACCGGTG avium type 4 CTGAACGAAAAGAACCTCGCCTTCCACCTGGGCGGCCACGTCAACCACTCGATCTGGTGGAAGAACCTGTCGCCGGACGGCGGTGACAAGCCCACCGGCG avlum type 5 CTGAACGAAAAGAACCTCGCCTTCCACCTGGGCGGCCACGTCAACCACTCGATCTGGTGGAAGAACCTGTCGCCGGACGGCGGTGACAAGCCCACCGGCG celat um t 3 TTGAACGAGAAGAACCTGGCGTTCAACCTGGCCGGCCACGTGAACCACACCATCTGGTGGAAGAACCTGTCGCCTAACGGCGGTGACAAGCCGACCGGTG celatum t 2 TTGAACGAGAAGAACTTGGCGTTCAACCTGGCCGGCCACGTCAACCACACCATCTGGTGGAAGAACCTGTCGCCCAACGGCGGTGACAAGCCGACCGGGG chelone1 CTCAACGAGAAGAACCTGGCCTTCCACCTCGGTGGCCACGTGAACCACTCCATCTGGTGGAAGAACCTGTCGCCCAACGGCGGCGACAAGCCCACCGGCG fort ult um CTCAACGAGAAGAACCTGGCGTTCCATCTCGGCGGCCACGTGAACCACTCGATCTGGTGGAAGAACCTGTCCCCCAACGGTGGCGACAAGCCGACGGGCG gordonae TTGAACGAGAAGAACCTGGCCTTCCACCTGGGCGGTCACGTCAACCACTCCATCTGGTGGAAGAACCTGTCGCCGGACGGCGGCGACAAGCCGACCGGTG int ra typel CTGAACGAAAAGAACCTGGCCTTTCACCTGGGCGGCCACGTCAACCACTCCATCTGGTGGAAGAACCTGTCGCCGGACGGCGGCGACAAGCCGACCGGCG intra type2 CTGAACGAAAAGAACCTGGCCTTTCACTTGGGCGGCCACGTCAACCACTCCATCTGGTGGAAGAACCTGTCGCCGGACGGCGGCGACAAGCCGACCGGCG int 1 t Int ra type 4 CTGAACGAAAAGACCTCGCATTCCACCTCGGTGGCCACGTCAACCACTCGATCTGGTGGAAGAACCTGTCGCCGGACGGCGGCGACAAGCCGACCGGCG int ra type 5 CTGAACGAAAAGAACCTCGCCTTCCACTTGGGCGGCCACGTCAACCACTCCATCTGGTGGAAGAACCTGTCGCCGGACGGCGGCGACAAGCCGACCGGCG malmoense marinum CTGAACGAAAAGAACCTCGCCTTCCACTTGGGCGGCCACGTCAACCACTCCATCTGGTGGAAGAACCTGTCGCCGGACGGCGGCGACAAGCCGACCGGCG CTGAACGAAAAGAACCTGGCCTTCCACCTGGGCGGGCACGTCAACCACTCGATCTGGTGGAAGAACCTGTCACCGAATGGCGGCGACAAGCCCACCGGCG CTGAACGAGAAGAACCTCGCTTTCAACCTCGCCGGCCACGTCAACCACACCATCTGGTGGAAGAACCTCTCCCCCAACGGTGGCGACAAGCCCACCGGTG $k$ ansas 11 t 1 CTGAACGAGAAGAACTTGGCCTTCAACCTCGCCGGCCACGTCAACCACACGATCTGGTGGAAGAACCTTTCTCCCAACGGAGGGGACAAGCCGACCGGCG kansas 11 t 2 CTGAACGAGAAGAACTTGGCCTTCAACCTCGCCGGCCACGTCAACCACACGATCTGGTGGAAGAACCTTTCTCCCAACGGAGGCGACAAGCCGACCGGCG paratb CTGAACGAAAAGACCTCGCCTTCCACCTGGGCGGCCACGTCAACCACTCGATCTGGTGGAAGAACCTGTCGCCGGACGGCGGTGACAAGCCCACCGGCG phle1 CTGCACGAGAAGAACCTTGCGTTCCACCTCGGCGGGCACGTCAACCACACGATCTGGTGGAAGAACCTGTCCCCGCACGGTGGCGACAAGCCGACCGGGG M.sp. 40407 CTGAACGAAAAGAACTTGGCGTTCAACCTGGCGGGCCACGTCAACCACAGCCTGTGGTGGAAGAACCTGTCGCCCGACGGTGGCGACAAGCCGACCGGCG scrof $t 1$ CTGAACGAAAAGAACCTGGCGTTCCACCTCGGCGGCCACGTGAACCACTCGATCTGGTGGAAGAACCTCTCGCCGGACGGCGGCGACAAGCCGACCGGAG scrof t 2 CTGAACGAGAAGAACTGGCGTTCCACCTCGGCGGCCACGTGAACCATTCCATCTGGTGGAAGAACCTGTCGCCGGACGGCGGCGACAAGCCGACCGGAG scrof t 2 CTGAACGAGAAGAACCTGGCGTTCCACCTCGGCGGCCACGTGAACCATTCCATCTGGTGGAAGAACCTGTCGCCGGACGGCGGCGACAAGCCGACCGGAG
s $1 \mathrm{~m} 1 \mathrm{ae}$ $\begin{array}{ll}\mathrm{s} 1 \mathrm{~m} 1 \mathrm{ae} & \text { TTGAACGAGAAGAACCTGGCATTCCACCTCGGTGGCCACGTCAACCACTCCATCTGGTGGAAAAACCTGTCCCCGAACGGCGGAGACAAGCCGACCGGAG } \\ \text { smegmat is } & \text { CTGAACGAGAAGAACCTCGCGTTCCATCTCGGTGGCCACATCAACCACTCGATCTGGTGGAAGAACCTCTCCCCCAACGGTGGCGACAAGCCCACCGGCG }\end{array}$ $\begin{array}{ll}\text { smegmat is } & \text { CTGAACGAGAAGAACCTCGCGTTCCATCTCGGTGGCCACATCAACCACTCGATCTGGTGGAAGAACCTCTCCCCCAACGGTGGCGACAAGCCCACCGGCG } \\ \text { szulgal } & \text { CTGAGCGAAAAGAACCTGGCGTTCAACTTGGCCGGCCACGTCAATCACACCATCTGGTGGAAGAATCTGTCTCCCAACGGTGGTGACAAGCCAACGGGCG }\end{array}$ tb CTGAACGAAAAGAATCTAGCTTTCAACCTCGCCGGCCACGTCAATCACACCATCTGGTGGAAGAACCTGTCGCCTAACGGTGGTGACAAGCCCACCGGCG GGGCATGAGAAGGCCCTCGCGTTCAACCTGGCCGGCCATGTCAATCACTGCCTGTGGTGGAAGAACCTGTCCCCCAACGGCGGTGACAAGCCGACCGGCG
393

human AGTTGCTGGAAGCCATCAAACGTGACTTTGGTTCCTTTGACAAGTTTAAGGAGAAGCTGACGGCTGCATCTGTTGGTGTCCAAGGCTCAGGTTGGGGTTG avium type 1 AGCTGGCCGCCGCGATCGACGACGCGTTCGGGTCCTTCGACAAGTTCCGAGCGCAATTCAGCGCCGCCGCCAACGGCCTGCAGGGCTCCGGCTGGGCGGT avium type 2 AGCTGGCCGCCGCGATCGACGACGCGTTCGGGTCCTTCGACAAGTTCCGAGCGCAATTCAGCGCCGCCGCCAACGGCCTGCAGGGCTCCGGCTGGGCGGT avium type 3 AGCTGGCCGCCGCGATCGACGACGCGTTCGGGTCCTTCGACAAGTTCCGAGCGCAATTCAGCGCCGCCGCCAACGGCCTGCAGGGCTCCGGCTGGGCGGT avium type 4 AGCTGGCCGCCGCGATCGACGACGCGTTCGGGTCCTTCGACAAGTTCCGGGCGCAATTCAGCGCCGCCGCCAACGGCCTGCAGGGCTCCGGCTGGGCGGT avium types AGCTGGCCGCCGCGATCGACGACGCGTTCGGCTCCTTCGACAAGTTCCGTGCGCAATTCAGCGCCGCCGCCAACGGACTGCAGGGCTCCGGCTGGGCGGT celatum t3 AGCTGGCCGCGGCCATCGACGACGCGTTCGGCTCGTTCGACAAATTCCGCGCTCAGTTCCACGCCGCCGCCACCACGGTGCAGGGGTCGGGATGGGCAGC celatum t2 AGCTGGCCGCGGCCATCGACGACGCGTTCGGCTCGTTCGACAAATTCCGCGCTCAGTTCCATGCGGCCGCCACCACCGTGCAGGGGTCGGGTTGGGCGGC chelone 1 ATCTGGCCGCCGCCATCGACGACCAGTTCGGCTCGTTCGACAAGTTCCAGGCACAGTTCACCGCCGCGGCCAACGGCCTGCAGGGCTCGGGCTGGGCCGT fortult um ACCTGGCCGCGGCCATCGACGACCAGTTCGGCTCGTTCGACAAGTTCCAGGCGCAGTTCACCGCCGCCGCCAACGGGCTGCAAGGCTCCGGCTGGGCCGT gordonae ATCTGGCCGCCGCGATCGACGACCAGTTCGGCTCGTTCGACAAGTTCCAGGCTCAGTTCAGCGCCGCCGCAAACGGCCTACAGGGCTCGGGCTGGGCGGT int ra typel AATTGGCCGCCGCGATCGACGACGCCTTCGGATCCTTCGACCGGTTCCGCGCGCAGTTCAGCGCGGCCGCCAACGGCCTGCAGGGGTCGGGCTGGGCGGT int ra type 2 AATTGGCCGCCACGATCGATGACGCCTTCGGATCCTTCGACCGGTTCCGCGCGCAGTTCAACGCGGCCGCCAACGGCCTGCAGGGGTCGGGCTGGGCGGT int ra type 3 AATTGGCCGCCACGATCGACGACGCCTTCGGATCCTTCGACCGGTTCCGCGCGCAGTTCAGCGCGGCCGCCAACGGCCTGCAGGGGTCGGGCTGGGCGGT int ra type 4 AACTGGCTGCTGCGATCGACGACGCGTTCGGGTCCTTCGACCGATTCCGCGCGCAATTCAGCGCGGCCGCCAACGGGCTGCAGGGCTCGGGCTGGGCCGT int ra type 5 AACTGGCCGCCGCGATCGATGACGCCTTCGGCTCCTTCGACCGCTTCCGCGCTCAGTTCAGCGCGGCCGCCAACGGACTGCAGGGTTCGGGCTGGGCGGT int ra types AACTGGCCGCCGCGATCGATGACGCCTTCGGCTCCTTCGACCGCTTCCGCGCTCAGTTCAGCGCGGCCGCCAACGGACTGCAGGGTTCGGGCTGGGCGGT
leprae malmoense $\begin{array}{ll}\text { marl num } & \text { AACTCGCCGCGGCCATCGATGAGGCGTTCGGGTCGTTCGACAAGTTTCGGGCCCAGTTCCACGCCGCCGCGACCACCGTGCAGGGGTCGGGTTGGGCAGC } \\ \text { kansasil t } 1 \text { AACTCGCCGCGGCCATCGACGAGGCGTTCGGGTCCTTCGACAAGTTTCGTGTCCAATTCCACGCCGCCGCCACCACGGTGCAGGGGTCGTCTGGGCGC }\end{array}$ GGCTAGCCACTGACATTGATGAAACGTTTGGGTCGTTCGACAAATTCCGCGCTCAATTCAGCGCGGCCGCCAATGGCCTGCAAGGGTCAGGTTGGGCAGT ACCTCGCGTCCGCGATCGACGACGCGTTCGGATCGTTCGACAAATTCCGCGCCCAGTTCAGCGCGGCCGCCAACGGCTTGCAGGGATCGGGCTGGGCGGT kansas 11 t 2 AACTCGCCGCGGCCATCGACGAGGCGTTCGGGTCCTTCGACAAGTTTCGTGTCCAGTTCCACGCCGCCGCCACCACGGTGCAGGGGTCGGGCTGGGCGGC paratb AGCTGGCCGCCGCGATCGACGACGCGTTCGGCTCCTTCGACAAGTTCCGGGCGCAATTCAGCGCCGCCGCCAACGGCCTGCAGGGCTCCGGCTGGGCGGT phle1 ATCTCGCGGCTGCGATCGACGACCAGTTCGGATCGTTCGACAACTTTCGCGCGCAGTTCACCGCGGCCGCCAACGGTTTGCAGGGGTCCGGCTGGGCGGT M.sp.40407 AGCTCGCCGCCGCCATCGACGACGCGTTCGGGTCGTTCGACAAGTTCCGCGCCCAGTTCACGGCGGCGGCCACCACCGTGCAGGGGTCGGGGTGGGCGGC $\begin{array}{ll}\text { scrof } & t 1 \\ \text { scrof } & \text { A } 2 \text { ACTGGCCGCCGCGATCGATGACGCGTTCGGATCGTTCGACAAATTCCGCGCCCAGTTCAGTGCGGCCGCCAACGGCCTGCAGGGTTCGGGCTGGGCGGT }\end{array}$ $\begin{array}{ll}\text { scrof th } & \text { AGCTGGCCGCCGCGATCGACGACGCGTTCGGATCGTTCGACAAGTTCCGCGCGCAGTTCAGTGCGGCCGCCAACGGCCTGCAGGGTTCGGGCTGGGCGGT } \\ \text { simiae } & \text { ATCTCGCCGCCGCCATCGACGACGCCTTCGTTCGTTCGACAAGTTCCGCGCACAGTTCAGCGCCGCGCCAACGGCTTGCAGGGCTCGGGCTGGGCGGT }\end{array}$ smegmat is szulga 1 ATCTCGCCGCCGCCATCGACGACGCCTTCGGTTCGTTCGACAAGTTCCGCGCACAGTTCAGCGCCGCCGCCAACGGCTTGCAGGGCTCGGGCTGGGCGGT tb AGCTGGCCGCGGCCATCGATGACGCGTTCGGTTCATTCGACACGTTCAGTGCGCAGTTCCACGCCGCCTCCACCACCGTGCAGGTTCGGGCTGGGCAGT xenop1 AACTCGCCGCAGCCATCGCCGACGCGTTCGGTTCGTTCGACAAGTTCCGTGCGCAGTTCCACGCGGCCGCTACCACCGTGCAGGGGTCGGGCTGGGCGGC

necessary. Ten isolates identified originally as MAC gave an identical unique profile which we have reported elsewhere ${ }^{14}$ as $M$ celatum type
3. Isolates of $M$ scrofulaceum and $M$ kansasii both had two 16SrRNA profiles, identical across the species specific region, but differing 
493

human GCTTGGTTTCAATAAGGAACGGGACACTTACAAATTGCTGCTTGTCCAAATC-AGGATCCACTG--CAAGGAACAAC-AGGCCTTATTCCACTGCTGGG avium type1 GCTGGGCTATGACAC----CCTGGGCA---GCCGGTTGCTGACCTTCCAGCTCTACGACCAGCAGGCCAACGTCCCGCTGGGCATCATCCCGCTGCTGCA avium type2 GCTGGGCTATGACAC----CCTGGACA---GCCGGTTGCTGACCTTCCAGCTCTACGACCAGCAGGCCAACGTCCCGCTGGGCATCATCCCGCTGCTGCA avlum type 3 GCTGGGCTATGACAC----CCTGGGCA---GCCGGTTGCTGACCTTCCAGCTCTACGACCAGCAGGCCAACGTCCCGCTGGGCATCATCCCGCTGCTGCA avlum type4 GCTGGGTTATGACAC---CCTGGGCA---GCCGGTTGCTGACCTTCCAGCTCTACGACCAGCAGGCCAACGTCCCGCTGGGCATCATCCCGCTGCTGCA avium type5 GCTGGGTTATGACAC----CCTGGGCA---GCCGGTTGCTGACCTTCCAGCTCTACGACCAGCAGGCCAACGTCCCGCTGGGCATCATCCCGCTGCTGCA celatum t 3 ACTCGGCTGGGACAA----CCTCGGCG---AGAAGCTGCTGATCTTCCAGGTGTACGACCACCAGACCAACTTCCCGCTCGGCGTCGTCCCGCTGCTGC

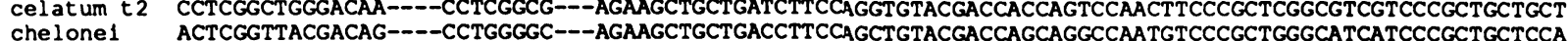
$\begin{array}{ll}\text { chelone1 } & \text { ACTCGGTTACGACAG----CCTGGGGC---AGAAGCTGCTGACCTTCCAGCTGTACGACCAGCAGGCCAATGTCCCGCTGGGCATCATCCCGCTGCTCCA } \\ \text { fortuitum } & \text { GCTCGGCTACGACAG----CCTGGGCG---ATCGGCTGCTGACCTTCCAGCTCTACGACCAGCAGGCAACGTGCCGTCGGCATCATCCCGCTGCTCCA }\end{array}$ $\begin{array}{ll}\text { fort uit um } & \text { GCTCGGCTACGACAG----CCTGGGCG---ATCGGCTGCTGACCTTCCAGCTCTACGACCAGCAGGCCAACGTGCCGCTCGGCATCATCCCGCTGCTCCA } \\ \text { gordonae } & \text { GCTCGGCTACGACAC----TCTGGGC---GCCGGTTGCTCACCTTCCAGCTCTACGACCAGCAGGCCAATGTCCGCTCGGTGTCATTCCGCTGTTGCA }\end{array}$ gordonae GCTCGGCTACGACAC----TCTGGGCG---GCCGGTTGCTCACCTTCCAGCTCTACGACCAGCAGGCCAATGTCCCGCTCGGTGTCATTCCGCTGTTGCA int ra typel GCTGGGCTACGACAC----CCTCGGCA---ACCGGCTGCTGACCTTCCAGCTCTACGACCAGCAGGCCAACGTGCCGCTGGGCATCATTCCGCTGCTGCA int ra type2 GTTGGGCTACGACAC----CCTCGGCA---GCCGGCTGCTGACCTTCCAGCTCTACGACCAGCAGGCCAACGTGCCGCTGGGCATCATTCCGCTGCTGCA int ra type 3 GCTGGGCTACGACAC----CCTCGGCA---ACCGGCTGCTGACCTTCCAGCTCTACGACCAGCAGGCCAACGTGCCGCTGGGCATCATTCCGCTGCTGCA Int ra type4 CCTGGGCTACGACAC----CCTCGGCA---ACCGGCTGCTGACCTTCCAGCTGTACGACCAGCAGGCCAACGTTCCGCTCGGCATCATCCCCTTGCTGCA Int ra type5 CCTGGGTTACGACAC----CCTGGGCA---GCCGGCTGCTGACCTTCCAGCTCTACGACCAGCAGGCCAACGTTCCGCTTGGCATCATCCCCTTGCTGCA leprae ACTCGGCTACGACAC---GCTCGGCA---ACAAGCTGCTTACATTCCAGCTGTATGACCAACAGGCCAACGTCTCACTCGGCATCATTCCCTTGTTGCA malmoense GCTCGGCTATGACTC----GCTCGGCG---ACAAGCTGCTGACGTTCCAGCTCTACGACCAGCAGGCCAACGTCCCGCTCGGCATCATTCCGCTGCTGCA marlnum
GCTGGGCTGGGACAC----CCTCGGCA---ACAAGCTGCTGATCTTCCAGGTCTACGACCACCAGACCAACTTCCCGCTGGGCATTGTCCCGCTGCTGCT

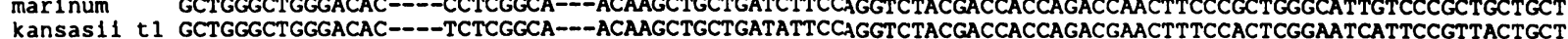
$\begin{array}{lll}k a n s a s & t 1 & \text { t } \\ \text { kansas } 11 & \text { t } 2 & \text { GCTTGGCTGGGACAC----TCTCGGCA---ACAAGCTCCTGATATTCCAGGTCTACGACCACCAGACGAACTTTCCGCTCGGAATCATTCCGTTACTGCT }\end{array}$ kansasil t 2 GCTTGGCTGGGACAC----TCTCGGCA---ACAAGCTCCTGATATTCCAGGTCTACGACCACCAGACGAACTTTCCGCTCGGAATCATTCCGTTACTGCT
paratb $\begin{array}{ll}\text { paratb } & \text { GCTGGGTTATGACAC----CGTGGGCA---GCCGGTTGCTGACCTTCCAGCTCTACGACCAGCAGGCCAACGTCCCGCTGGGCATCATCCCGCTGCTGCA } \\ \text { phle1 } & \text { GTTGGGCTACGACAC----CCTCGGT---ACCGGCTGCTGACCTTCCAGTTGTACGACCAGCAAGCAAATGTCCACTGGGGATCATCCCGCTGTTGCT }\end{array}$ phle1 10407 ACTCGGCTACGACAC----CCTCGGTG---ACCGGCTGCTGACCTTCCAGTTGTACGACCAGCAAGCAAATGTCCCACTGGGGATCATCCCGCTGTTGCT M.sp. 40407 ACTCGGCTGGGACAG----CCTCGGCG---AGAAGTTGCTGGTGTTCCAGGTGTACGACCACCAGACGAACTTCCCGCTCGGCATCGTCCCGTTGCTGGT
scrof $\mathrm{t} 1 \mathrm{GCTGGGCTATGACAC---GCTCGGC---GCAGGCTGCTCACCTTCCAGCTTACGACCAGCAGGCCAACGTCCCGCTCGGCATCATTCCGCTGCTGCA}$ scrof $t 2$ GCTGGGCTACGACAC---CCTCGGCA---GCAGGCTGCTCACCTTCCAGCTTTACGACCAGCAGGCGACGTCCCCCTCGGCATCATTCCGCTGCTGCA simiae ACTCGGCTACGACAC----CCTCGGCG---ACCGACTGCTGACCTTCCAGCTTTACGACCAGCAGGCCAACGTCCCGCTGGGCATCATCCCGCTGCTGCA smegmat is GCTCGGATACGACAG----CCTCGGTG---GCCGTCTGCTGACCTTCCIGCTCTACGACCAGCAGGCCAACGTGCCGCTCGGCATCATCCCGCTCCTCCA smegmat is GCTCGGATACAG-CCICGGT-OCCGTCTGCTGACCTTCCAGCTCTACGACCAGCAGGCCAACGTGCCGCTCGGCATCATCCCGCTGCTCCA szulga1 ACTGGGATGGGACAC----ACTGGGTA---ACAAGCTGCTGATCTTCCAGGTCTACGACCACCAGACCAACTTCCCCCTCGGCATCGTTCCGTTACTGCT tb 1 ACTGGGCTGGGACAC----ACTCGGCA---ACAAGCTGCTGATATTCCAGGTTTACGACCACCAGACGAACTTCCCGCTAGGCATTGTTCCGCTGCTGCT xenopi $\quad$ ACT

human GATTGATGTGTGGGAGCACGC

avium typel GGTCGACA

avium type2 GGTCGACATGTGGGAGCACGC

avium type 3 GGTCGACATGTGGGAGCACGC

avium type4 GGTCGACATGTGGGAGCACGC

avium type5 GGTCGACATGTGGGAGCACGC

celatum t 3 CCTCGACATGTGGGAGCACGC

celatum t2 CCTCGACATGTGGGAGCACGC

chelone1 GGTCGACATGTGGGAGCACGC

chelone GGTACATGAGCACGC

GGTCGACATTGGGAGCACGC

gordonae GGTCGACATGTGGGAGCACAC

ntrat 1 GGTCGACATGTGGGAGCACGC

intra t2 GGTCGACATGTGGGAGCACGC

Intra t3 GGTCGACATGTGGGAGCACGC

intra t4 GGTCGACATGTGGGAGCACGC

intra t5 GGTCGACATGTGGGAGCACGC

leprae GGTCGACATGTGGGAACATGC

malmoense GGTCGACATGTGGGAACACGC

marinum GCTCGACATGTGGGAGCACGC

kansas 11 t 1 GCTCGACATGTGGGAACACGC

kansas 11 to GCTCGACATGTGGGAACACGC

kanstl t2 GCTCGACATCTGGGAACACGC

paratb GGTCACATGTGGAGCACGC

phlel 40407 GGTCGACATGTGGGAACATGC

M.sp. 40407

GGTCGACATGTGGGAGCACGC

scrof t2 GGTCGACATGTGGGAGCACGC

simiae GGTCGACATGTGGGAGCACGC

smegmat is GGTCGACATGTGGGAGCACGC

szulga1 GCTCGACATGTGGGAACACGC

tb GCTCGACATGTGGGAACACGC

Figure 1 Alignment of superoxide dismutase gene sequences.

\section{SOD SEQUENCES}

All 104 isolates gave a product with the primers SF1 and SR1 which were sequenced in both directions at least once (fig 1 ). Five sequence types of $M$ avium were observed which showed only small variations (fig 2 ) in base sequence $(0.9$ (SD 0.4$) \%)$. Thirty eight isolates referred to here as $M$ avium type 1 included 33 AIDS, five non-AIDS, and the reference strain NCTC 8559. Four further isolates each had unique sequence variations and are referred to here as $M$ avium types 2 and 3 (AIDS isolates), and $M$ avium types 4 and 5 (non-AIDS isolates).
AIDS isolates of $M$ avium could not be distinguished from non-AIDS isolates on these sequence data. Isolates identified by $16 \mathrm{SrRNA}$ sequencing as similar to $M$ intracellulare could be differentiated by minor base variations into five types, referred to as follows: three isolates designated $M$ intracellulare type 1 (one AIDS isolate, one non-AIDS isolate, and reference strain NCTC 10682); one isolate designated $M$ intracellulare type 2 (AIDS isolate); three isolates designated $M$ intracellulare type 3 (AIDS isolates concurrent with $M$ intracellulare serotype $1816 \mathrm{SrRNA}$ profile); three isolates designated $M$ intracellulare type 4 (AIDS isolates with previously unreported $16 \mathrm{SrRNA}$ profile); and one isolate designated $M$ intracellulare type 5 (reference strain $M$ intracellulare serotype 7; ATCC 35847). Similarly $M$ scrofulaceum had one isolate designated $M$ scrofulaceum type 1 (reference strain NCTC 10803) and one isolate designated $M$ scrofulaceum type 2 (AIDS isolate). Overall the MAC isolates varied by $7 \cdot 1$ $(3.7) \%$ and were distinctly clustered in the calculated phylogenetic tree (fig 3 ) with a 19.5 $(4 \cdot 1) \%$ diversity of this group from all other 


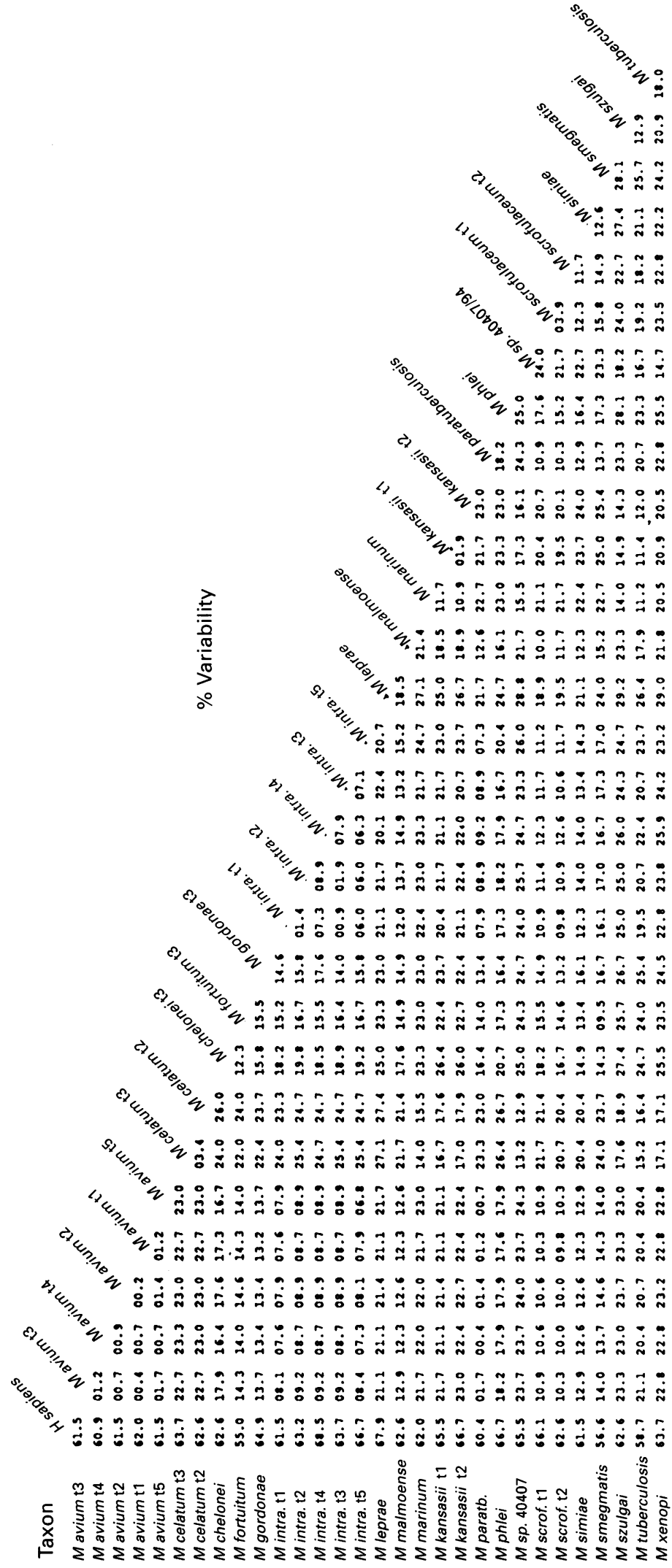

Figure 2 Superoxide dismutase gene variability matrix calculated with fukes and Cantor distance method. ${ }^{12}$ minor 16SrRNA sequence variation). $M c e-$ latum type 1 and 10 isolates of $M$ celatum type 3 showed identical sequences but had a $3 \cdot 5 \%$ variation from $M$ celatum type 2 and 21.5 (3.7)\% variation from all other species. All 11 isolates of $M$ tuberculosis had identical sequences. Significant variation (range: 10-29) was observed between all the remaining reference strains, with all the rapid growing species also forming a distinct cluster which had $18 \cdot 6$ $(4 \cdot 0) \%$ diversity from all slow growing species. Restriction digest maps produced from each of the 29 sequence variations revealed that HaeIII would be a suitable enzyme for the generation of unique restriction fragment profiles.

\section{CAPILLARY ELECTROPHORESIS PROFILES}

Capillary electrophoresis of samples gave chromatograms for the fragment size range 50-264 bp. The inclusion of a standard digest into each capillary electrophoresis sample allowed an estimation of sample fragment sizes (fig 4). Capillary electrophoresis profiles, in the range 70-264 bp, were plotted for at least one representative of all isolates with unique SOD sequences. Twenty unique profiles were determined (table) which could differentiate all the recognised species studied with the exceptions of $M$ avium from $M$ paratuberculosis, and $M$ celatum types 1 and 3 from $M$ szulgai. All internal fragment sizes corresponded to those predicted from the sequence data. The majority of terminal fragments, however, gave estimated sizes of 3-7 bases larger than expected. These increases were constant for each fragment length and were the same for all species and repeat samples tested. In some samples, extra peaks were observed at $57 \mathrm{bp}$ and $60 \mathrm{bp}$ (fig 4). These peaks could not be explained from the sequence data and may have occurred because of non-specific amplification or primer concatenation. Their appearance also varied from sample to sample and did not occur in all repeat samples. These peaks, however, were below the chosen capillary electrophoresis profile range and therefore did not interfere with the differentiation of isolates.

NUCLEOTIDE SEQUENCE ACCESSION NUMBERS The following sequences have been submitted to EMBL (European Molecular Bank Library) under the accession numbers: Z48208 ( $\mathrm{M} \mathrm{ma}$ rinum), Z48209 (M celatum type 3 ), Z48210 (M malmoense), Z48211 (M phlei), Z48212 (M paratuberculosis), Z48213 (M szulgai), Z48214 (M smegmatis), Z48215 (M celatum type 3 ), Z48216 (M chelonei), Z48217 (M intracellulare serotype 18), and Z48218 (M intracellulare serotype 7$)$. Other sequences in this work were found to be identical to the following existing sequences: X52861 ( $M$ tuberculosis), X16453 (M leprae), X81384 (M avium), X81387 ( $M$ intracellulare), X81385 ( $M$ fortuitum), X81386 ( $M$ gordonae), $\mathrm{X} 81388$ ( $M$ kansasii), and X81390 (M simiae). species. $M$ kansasii had 12 isolates designated $M$ kansasii type 1 -including nine AIDS isolates, two non-AIDS isolates, and the reference strain NCTC 10268-and one isolate designated $M$ kansasii type 2 (AIDS isolate with

HOH




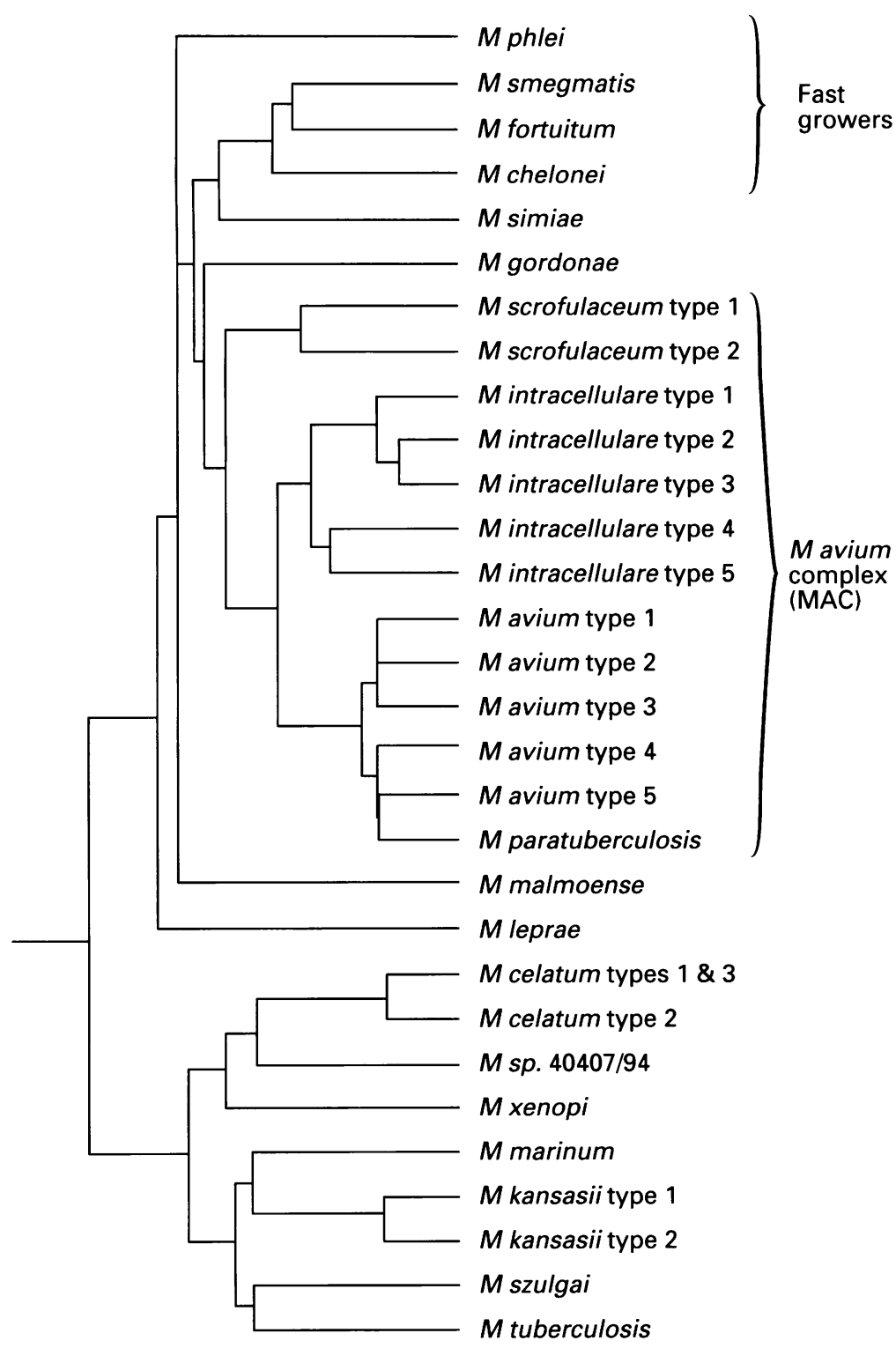

Figure 3 Phylogenetic tree of mycobacteria derived from superoxide dismutase (SOD) gene by FITCH algorithm using $\mathrm{H}$ sapiens $S O D^{13}$ as outgroup.

\section{Discussion}

The diversity shown by the sequences presented in this study indicates that the SOD gene is suitable as an alternative to genes such as 16SrRNA for the genomic identification of the Mycobacteriaceae. AIDS isolates of $M$ avium could not be distinguished from non-AIDS isolates; however, a phenogram derived from SOD sequences (fig 3) clearly delineates fast and slow growing species and MAC. $M$ avium, $M$ intracellulare, and $M$ scrofulaceum are seen to form a cluster representative of MAC which does not include $M$ celatum as suggested by biochemical identifications alone. ${ }^{15}$ The phylogenetic diversity of the $M$ celatum group from MAC (23.3 (1.3)\%) which had been previously suggested using $16 \mathrm{SrRNA}$ data is thus confirmed, with $M$ celatum being more related to $M$ xenopi than MAC. All recognised species used in this study were differentiated by $8-29 \%$ base variation with the exception of $M$ paratuberculosis, which differed from the observed five $M$ avium types by up to four bases $(1 \cdot 1$ $(0.5) \%)$. This difference could be significant considering the close genetic homology already recognised in studies of other genes from these two species, and that the variation at base No 513 (fig 1) predicts a change in amino acid sequence (valine to leucine). These data concur with 16 SrRNA studies in that $M$ paratuberculosis should be regarded as a subtype of $M$ avium. We believe this report is the first to be able to differentiate $M$ paratuberculosis from $M$ avium by gene sequence data alone. Minor intraspecies sequence differences were also observed between clinical isolates and reference strains within the species of $M$ scrofulaceum (3.9\%), $M$ kansasii $(1.9 \%)$, and $M$ celatum (3.5\%). In the $M$ intracellulare samples a larger variation of $5 \cdot 4(2 \cdot 7) \%$ was observed, illustrating the high diversity within this loosely defined species.

We show here that sequence diversity in SOD can be used to predict unique capillary electrophoresis profiles and have rapidly differentiated 16 of 18 species tested with this method. We have only used a single restriction enzyme digest in this study; however, in combination with further restriction digests it would be possible to differentiate all the species described. The advantage of using a single low cut restriction digest can be seen with the results from $M$ avium and $M$ kansasii, where intraspecies base variation did not occur across restriction sites and thus only one unique profile was seen for all the species types (table).

Terminal restriction digest fragment sizes observed by capillary electrophoresis in the majority of species were constantly larger than predicted from the sequence data. Similar migration retardation anomalies have been reported, ${ }^{16}$ possibly due to DNA fragment secondary structure and the concentration and type of polymer used in the capillary. The reproducibility of the technique described here suggests, however, that the observed larger fragment sizes will not present a problem in the identification of species in unknown samples, providing adequate control profiles are used.

A recently described method ${ }^{17}$ also uses the SOD gene to detect mycobacterial speciesspecific sequences. This method presupposes that intraspecies diversity is low enough to allow relatively high stringency hybridisation of short species-specific DNA probes homologous to amplified SOD product. This study shows that four of $42(10 \%)$ clinical isolates of $M$ avium contained sequence variations. If variation occurs within the probe sites it could result in failure to hybridise, thereby reducing the specificity of the test. Our approach using identification by low cut restriction analysis circumvents this potential problem by producing capillary electrophoresis profiles for all isolates, regardless of sequence variation. The use of a low cut enzyme guarantees a low probability of variation across restriction sites which would produce profiles dissimilar to the control profile bank. Even in this event, profiles can be extrapolated to give the most likely identification from the nearest fit. This study shows that the high diversity between SOD sequences in mycobacteria ensures that profiles of one species will not be transformed by minor 

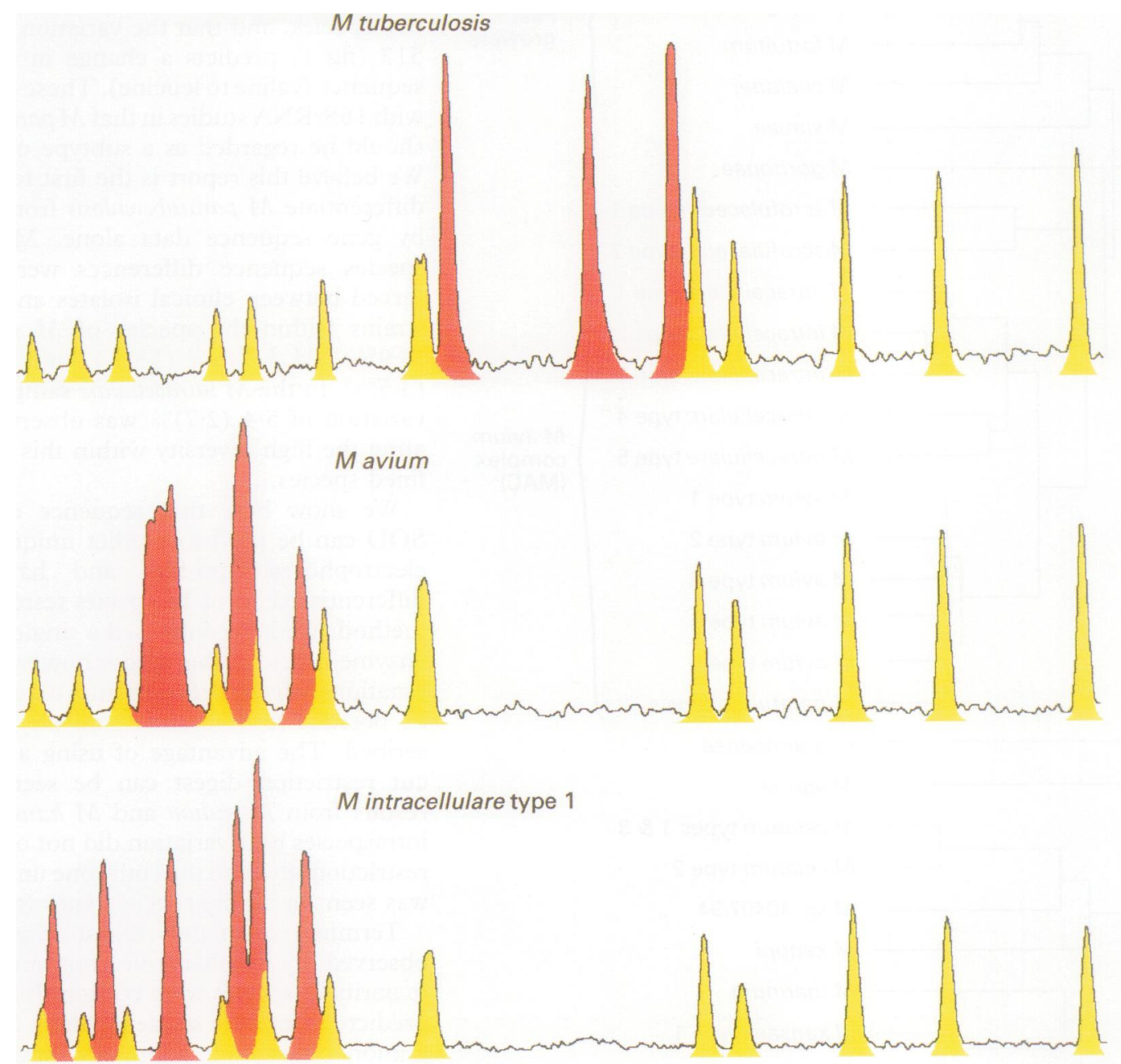

$M$ intracellulare type 4

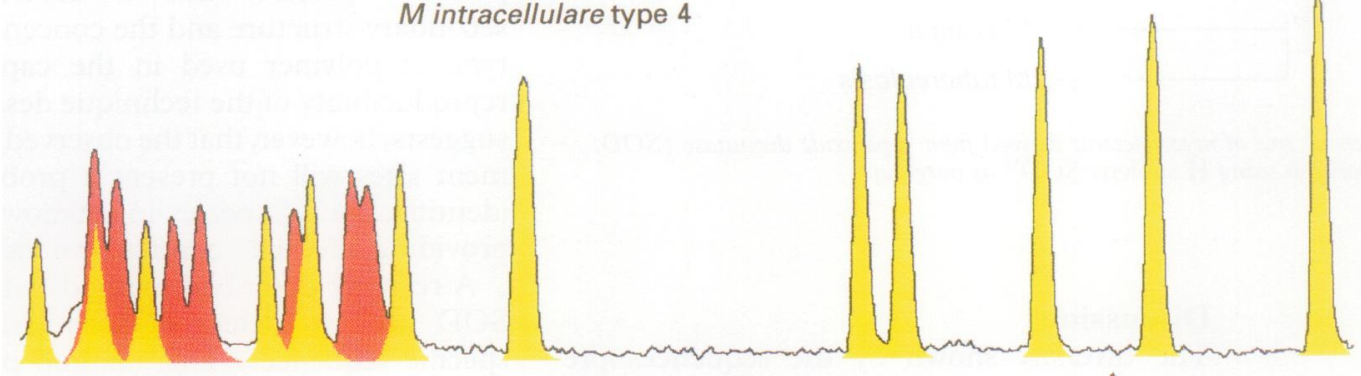

M celatum type 3

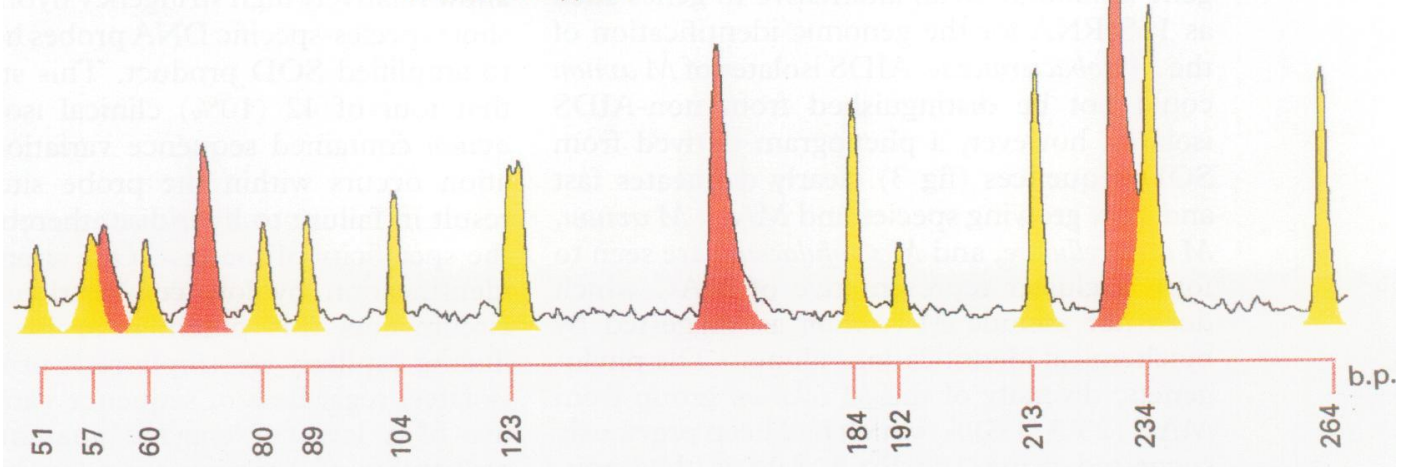

Sample fragments

Standard fragments (pBR322 Haelll digest)

Figure 4 Capillary electrophoresis profiles of amplified superoxide dismutase gene HaeIII restriction digests from mycobacterium species. 


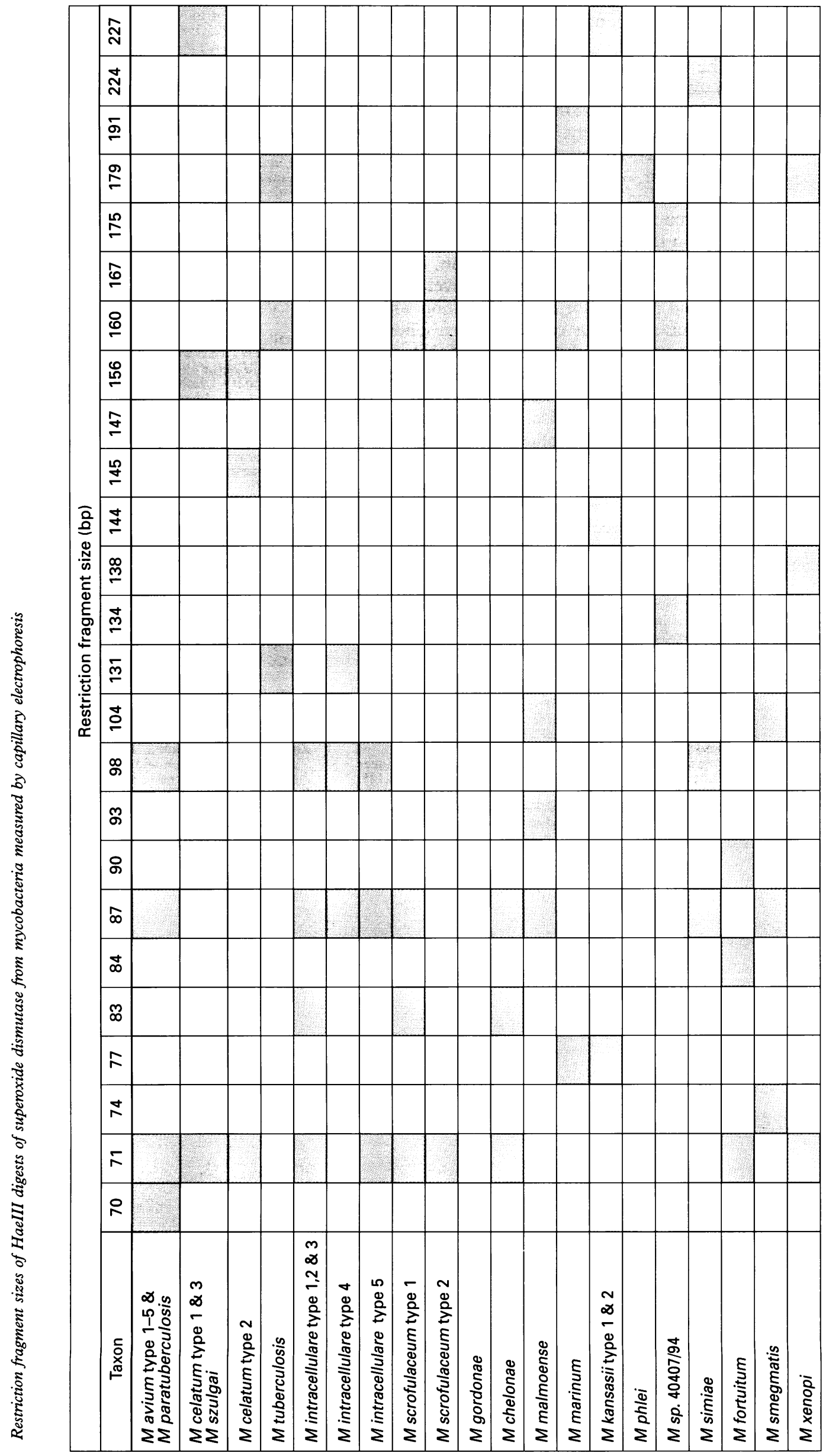


sequence variation into those corresponding to another species.

The need for rapid detection of small numbers of bacteria present in clinical samples requires an efficient DNA extraction technique to maintain sensitivity. The nature of the mycobacterial cell wall in species such as $M$ intracellulare and $M$ avium makes it difficult to achieve lysis efficiently without the use of long and technically demanding procedures. ${ }^{18}$ Rapid detection techniques have therefore tended to be concentrated on the more easily lysed $M$ tuberculosis. We have developed a novel, simple, and rapid method for DNA extraction which, in conjunction with capillary electrophoresis profile analysis of the amplified SOD gene, enables identifications to be carried out on all mycobacterial species within 24 hours. Preliminary work on clinical samples using extraction techniques and PCR conditions described here indicate that our SOD primers are genus specific. We therefore believe that this method will be useful for rapid differential diagnosis of mycobacteria when applied directly to clinical samples.

The authors would like to thank Mr M D Yates of the Regional Centre for Tuberculosis Bacteriology, Dulwich Hospital, London, for his help in providing and identifying biochemically some of the clinical isolates used in this study. This work also benefited from the use of the SEQNET facility (Network No 148.79.64.2 1)

1 Wayne LG, Krichevsky MI, Portyrata D, Jackson CK. Diagnostic probability matrix for identification of slow growing mycobacteria in clinical laboratories. $f$ Clin Microbiol ing mycobacteria

2 Crawford JT. Development of rapid techniques for identification of $M$. avium infections. Res Microbiol 1994;145 $177-80$

3 Van de Vliet GME, Schukkink RAF, Van Gemen B, Schepers $P$, Klatser PR. Nucleic acid sequence-based amplification
(NASBA) for the identification of mycobacteria. $f \mathrm{Gen}$ Microbiol 1993;139:2423-9.

4 White TJ, Madej R, Pershing DH. The polymerase chain reaction: clinical applications. Adv Clin Chem 1992;29: 161-96.

5 Bull TJ, Shanson DC. Rapid misdiagnosis by $\mathrm{Myco}$ bacterium avium-intracellulare masquerading as tuberculosis in PCR/DNA probe tests. Lancet 1992;340:1360.

$6 \mathrm{Masur} H$. US Public health service task force on prophylaxis and therapy for Mycobacterium avium complex. Recommendations on prophylaxis and therapy for disceminated Mycobacterium avium complex disease in patients with AIDS. N Engl 7 Med 1993;329:896-904.

7 Jacobson MA, Yajko D, Northfelt D, Charlebois E, Gary Jacobson MA, Yajko D, Northfelt D, Charlebois E, Gary
D, Brosgart C, et al. Randomised, placebo-controlled $\mathrm{D}$, Brosgart C, et al. Randomised, placebo-controlled
trial of rifampin, ethambutol and ciprofloxacin for AIDS trial of rifampin, ethambutol and ciprofloxacin for AIDS F Infect Dis 1993;168:112-9

8 Zhang Y, Lathigra R, Garbe T, Catty D, Young D. Genetic analysis of superoxide dismutase, the 23 kilodalton antigen of Mycobacterium tuberculosis. Mol Microbiol 1991;39:38191.

9 Rogall T, Flohr T, Böttger EC. Differentiation of Mycobacterium species by direct sequencing for amplified DNA. bacterium species by direct sequencin
7 Gen Microbiol 1990;136:1915-20.

10 Thangaraj HS, Lamb I, Davis EO, Jenner PJ, Jeyakumar LH, Colston MJ. Identification, sequencing and expression of Mycobacterium leprae superoxide dismutase, a major antigen. Infect Immun 1990;58:1937-42.

11 Thangaraj HS, Lamb FI, Davis EO, Colston MJ. Nucleotide and deduced amino acid sequence of Mycobacterium leprae manganese superoxide dismutase. Nucleic Acids Res 1989; 17:8378.

12 Jukes TH, Cantor CR. Evolution of protein molecules. In: Munro HN, ed. Mammalian protein metabolism, vol 3. New York: Academic Press, 1969;21-132.

13 Ho YS, Crapo JD. Isolation and characterization of complementary DNAs encoding human manganese-conplementary DNAs encoding human manganese-containing $256-60$.

14 Bull TJ, Shanson DC, Yates MD, Hamid ME, Minnikin DE. A new type of Mycobacterium celatum isolated from AIDS patients in the London area. Int $\mathcal{F}$ Syst Bacteriol (in press)

15 Butler WR, O'Connor SP, Yakrus MA, Smithwick RW, Plikaytis BB, Moss CW, et al. Mycobacterium celatum sp. nov. Int $\mathcal{F}$ Syst Bacteriol 1993;43:539-48.

16 Wenz HM. Capillary electrophoresis as a technique to analyse sequence-induced anomalously migrating DNA fraglyse sequence-induced anomalously migrating
ments. Nucleic Acids Res 1994;22:4002-8.

17 Zolg JW, Philippi-Schulz S. The superoxide dismutase gene, a target for detection and identification of mycobacteria by PCR. $\mathcal{f}$ Clin Microbiol 1994;32:2801-12.

18 Rastogi N, Barrow WW. Cell envelope constituents and the multifaceted nature of Mycobacterium avium pathogenicity and drug resistance. Res Microbiol 1994;145:243-52. 